\title{
Editoriale
}

\section{Nefropatia da mezzo di contrasto: il parere del Radiologo}

\section{R. Carpi}

S.C. di Radiodiagnostica, Ospedale S. Maria Nuova, Azienda Sanitaria di Firenze

CONTRAST-INDUCED NEPHROPATHY: RADIOLOGIST'S OPINION

ABstRact. Contrast-induced nephropathy (CIN) is one of the most important cause of hospital-acquired acute kidney failure. The use of e-GFR instead of serum creatinine is more predictive for the development of CIN for those patient frequently affected by other pathologies (elderly patients with comorbidities) but it increases number of patient to be hydrated, side effect compensated by the lower $45 \mathrm{ml} / \mathrm{min}$ cut-off recently indicated in guidelines for those patients who will receive intravenous iodinated contrast media.

Hydration is widely considered the most effective prophylactic tool but it will be effectively implemented only if we will be able to develop necessary synergies between the prescribing physician, radiologist and nephrologist.

Key Words. CIN, Iodinated CONTRAST MEDIa, e-GFR

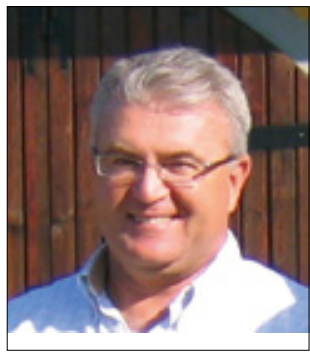

Roberto Carpi
La Nefropatia Indotta da mezzo di Contrasto (CIN) è una entità patologica definita come un incremento della creatinina plasmatica, assoluto o relativo, conseguente a una ridotta funzionalità renale, a sua volta dovuto al danno renale indotto dalla somministrazione endovascolare di mezzo di contrasto. Si manifesta raramente in popolazione con normale funzionalità renale pre-contrasto ed è invece tra le cause più frequenti di insufficienza renale acuta in ambiente ospedaliero (1).

Il danno renale causato dai mezzi di contrasto (MDC) è da alcuni autori solo "supposto" poiché da una revisione critica della letteratura, pochi sono gli studi che hanno una potenza statistica sufficiente a dimostrare tale nesso causale e in quei pochi (2) non è emersa una differenza statisticamente significativa tra il gruppo di pazienti sottoposti a somministrazione di MDC ed il gruppo di controllo; per tale motivo alcuni autori avanzano l'ipotesi di una possibile sovrastima dell'azione nefropatica dei MDC.

Resta comunque il dato di fatto che il sempre maggior ricorso all'utilizzo della diagnostica per immagini con la somministrazione di MDC, pone il problema del reale rischio di danno iatrogeno. Ruolo fondamentale nella pre- venzione della CIN viene giocato dalla identificazione dei pazienti a rischio, e tra i fattori di rischio di maggior importanza, la maggior parte degli studi pubblicati individua una preesistente insufficienza renale, in particolare se associata ad altre condizioni patologiche, prima fra tutte il diabete.

Nel corso degli ultimi anni si è ricorso sempre più frequentemente all'utilizzo di strumenti più "sofisticati" ma al contempo agili da utilizzare per discriminare meglio i pazienti potenzialmente a rischio di sviluppare una CIN; infatti utilizzare come cut-off il solo valore della creatinina sierica ha il limite intrinseco di sottostimare il rischio in una fascia di popolazione consistente e costituita da pazienti anziani, in particolare se di sesso femminile, in particolare se diabetici, con il conseguente rischio di sottoporre alla somministrazione del MDC un paziente che poi potrà sviluppare una $\mathrm{CIN}$, non sempre reversibile. Da qui l'importanza di aver introdotto il calcolo del filtrato glomerulare (e-GFR) mediante l'utilizzo di formule semplici di calcolo, tanto da poter essere automaticamente inserite nei referti laboratoristici, poiché basate solamente sul dosaggio della creatinina plasmatica, sul sesso, la razza e sull'età del paziente.

Passare dal cut-off di $1.5 \mathrm{mg} / \mathrm{dL}$ della creatinina plasmatica a quello di $60 \mathrm{~mL} / \mathrm{min}$, come inizialmente identificato, di e-GFR ha comportato certamente un incremento del numero di pazienti definibili potenzialmente 
"a rischio" CIN (3), ovvero del numero di pazienti che, incontrando i criteri definiti nelle diverse linee guida, necessitavano di un'azione profilattica mediante idratazione. E per quest'ultima sono stati proposti molti schemi di idratazione, con l'infusione endovenosa di soluzione fisiologica, di $\mathrm{Na}$ bicarbonato, di somministrazione di liquidi per os.

Tutte le linee guida internazionali (4) riconoscono nell'idratazione un ruolo centrale nella profilassi della CIN e questo pone il problema di riuscire a creare un sistema organizzativamente integrato ed efficace per poter intercettare i pazienti a rischio e somministrare loro la giusta profilassi. Se questo è più semplice da realizzare per i pazienti ospedalizzati, che possono essere gestiti nell'intero arco della giornata da personale medico o infermieristico, non altrettanto si può dire per i pazienti ambulatoriali, per i quali sarebbe auspicabile una integrazione tra medico richiedente, generalmente il Medico di Medicina Generale (MMG), il radiologo e il nefrologo. Infatti nella maggior parte dei casi il MMG prescrive l'esame radiologico contestualmente agli esami del sangue necessari alla sua effettuazione (creatinina plasmatica); i risultati sono successivamente sottoposti al radiologo al momento dell'accesso in radiologia per l'effettuazione dell'esame, troppo tardi per poter attuare tutte quelle misure necessarie in caso di pazienti "a rischio" (e-GFR anormale). Questo ovviamente comporta uno spreco di risorse, non potendo spesso effettuare l'indagine, con conseguenze facilmente comprensibili in termini di spreco economico e di evitabili allungamenti dei tempi di attesa per le prestazioni diagnostiche. Una integrazione efficace dovrebbe invece prevedere una stretta correlazione tra il medico richiedente e le strutture eroganti la prestazione stessa, con il contributo delle nefrologie dei vari presidi ospedalieri, magari istituendo dei percorsi di Day Hospital, finalizzati ad accogliere il paziente per le ore strettamente necessarie prima e dopo la somministrazione del MDC e poter effettuare l'idratazione e.v. come previsto dalle differenti linee guida. Idratazione che dovrebbe, in alternativa, essere effettuata all'interno delle radiologie, non sempre adeguatamente attrezzate in termini di spazi e risorse per questo tipo di attività "collaterale". Resta comunque imprescindibile l'interazione con il prescrittore che dovrebbe accertare le condizioni di fattibilità dell'esame o la necessità di inserire il paziente nel percorso protetto della idratazione, in un tempo anteriore rispetto all'accesso in radiologia.

\section{Riassunto}

La Nefropatia da Mezzo di Contrasto (CIN) rappresenta una della cause più frequenti di insufficienza renale acuta in ambiente ospedaliero.

L'introduzione del calcolo del filtrato glomerulare (eGFR) al posto del semplice dosaggio della creatinina plasmatica, se da un lato consente una miglior identificazione dei pazienti a rischio, in particolare allinterno della fascia di popolazione più spesso sottoposta ad indagini radiologiche (pazienti anziane con comorbidità), dall'altro accresce il numero dei pazienti da considerare a rischio e da sottoporre a profilassi. L'aver riportato al valore di $45 \mathrm{~mL} / \mathrm{min}$ dagli iniziali 60 il valore di cut-off in base al quale sottoporre il paziente a idratazione profilattica in caso di somministrazione di mezzo di contrasto per via endovenosa (la più frequentemente impiegata in ambito radiologico extrainterventistico), compensa tale incremento.

I numerosi studi in letteratura e le linee guida internazionali descrivono multipli fattori di rischio ma convergono nel riconoscere nell'idratazione del paziente uno strumento utile per la profilassi della insorgenza della CIN. Questa potrà essere attuata in modo efficace solamente se si svilupperanno le sinergie organizzative necessarie tra medico prescrittore, radiologo e nefrologo.

Parole Chiave. CIN, Mezzo di contrasto iodato, e-GFR

\section{Indirizzo degli Autori:}

Dr. Roberto Carpi

Piazza S. Maria Nuova 1

50100 Firenze

roberto.carpi@asf.toscana.it

\section{Bibliografia}

1. Nash K, Hafeez A, Hou S. Hospital-acquired renal insufficiency. Am J Kidney Dis 2002; 39: 930-6.

2. Rao Q, Newhouse JH. Risk of nephropathy after intravenous contrast: a critical literature analysis. Radiology 2006; 239: 392-7.

3. Herst BR, Schneider E, Poggio E, et al. Identifying Outpatients with Renal Insufficiency before Contrast-enhanced CT by Using Estimated Glomerular Filtration Rates versus Serum Creatinine Levels. Radiology 2008; 248: 108-13.

4. Stacul F, Van der Molen AJ, Reimer P, et al. Contrast induced nephropathy: updated ESUR Contrast Media Safety Committee guidelines. Eur Radiol 2011; 21: 2527-41. 